\title{
Crab giant pulses at low frequencies
}

\author{
R. Karuppusamy ${ }^{1,3}$, B. W. Stappers ${ }^{2,3,4}$, and K. J. Lee ${ }^{1}$ \\ 1 Max-Planck-Institut für Radioastronomie, Auf dem Hügel 69, Bonn, Germany \\ e-mail: ramesh@mpifr-bonn.mpg.de \\ 2 Jodrell Bank Centre for Astrophysics, School of Physics and Astronomy, The University of Manchester, Manchester M13 9PL, UK \\ e-mail: Ben.Stappers@manchester.ac.uk \\ 3 Sterrenkunde Instituut Anton Pannenkoek, University of Amsterdam, Kruislaan 403, Amsterdam, The Netherlands \\ ${ }^{4}$ Netherlands Institute for Radio Astronomy (ASTRON), Postbus 2, 7990 AA, Dwingeloo, The Netherlands
}

Received 8 July 2011 / Accepted 3 December 2011

\begin{abstract}
We report observations of the Crab pulsar at frequencies in the range of 110-180 MHz. The combination of coherent dedispersion and the narrow synthesised beam of the Westerbork Synthesis Radio Telescope resulted in a sensitive observation. Our improved sensitivity and resolution allow us to confirm the presence of a precursor to the interpulse at these frequencies. We also detected more than 1000 giant pulses and find that the interpulse precursor component shows no giant pulse emission. Therefore, we attribute it to a similar emission source as the precursor to the mainpulse. Together these precursors might be the normal emission seen from the majority of radio pulsars. From the dispersion-free giant pulses, we find that the emission rate is $\sim 10-20 \times 10^{-3} \mathrm{~s}^{-1}$ and the scatter timescale in the range of $\sim 1.5-5.6 \mathrm{~ms}$. We further find that the radio flux of the pulsar is $6-11 \mathrm{Jy}$ in this frequency range.
\end{abstract}

Key words. radiation mechanisms: non-thermal - scattering

\section{Introduction}

The Crab nebula and pulsar are amongst the most intensively studied objects on the sky. The discovery of the Crab pulsar (Staelin \& Reifenstein 1968) in the Crab nebula (identified as the remains of the supernova, SN 1054) was the first observational evidence for neutron stars being formed in supernova explosions. The Crab pulsar is a bright source of electromagnetic radiation and is detected at all observable wavelengths. At radio frequencies, the pulsar emission shows a variety of features of which the evolution of the average pulse profile with increasing radio frequency is readily noticed. For example, in addition to the main- and interpulse emission visible at most frequencies, other emission components appear at certain frequencies; the precursor emission component is visibile only at low frequencies (Rankin et al. 1970); the low-frequency component appears between $600-4800 \mathrm{MHz}$ and two high-frequency components in the $4000-8400 \mathrm{MHz}$ frequency range (Moffett \& Hankins 1996). In the latter work, it is also seen that no interpulse emission is present at $2700 \mathrm{MHz}$, and reappears slightly earlier in phase above $4700 \mathrm{MHz}$. An enigmatic feature in the radio emission from the pulsar is the intense radio bursts called giant pulses, which actually led to the discovery of the Crab pulsar (Staelin $\&$ Reifenstein 1968). The giant pulses were reported for the first time by Sutton et al. (1971) as "jumbo" pulses that formed a long tail in the single pulse intensity histogram. Soon after this work, the individual pulses from the Crab pulsar were extensively characterised and the power-law nature of the pulse energy distribution was firmly estalished (Argyle \& Gower 1972; Gower \& Argyle 1972).

Giant pulses are now defined as pulses with energy greater than ten times the average pulse energy and have widths very narrow compared to the average pulse emission (Knight 2007). As an example, narrow pulses on the order of 0.4 ns are observed at a frequency of $9 \mathrm{GHz}$ (Hankins \& Eilek 2007). However, at low radio frequencies, the signal propagation effects in the interstellar medium (ISM) blur the narrow pulses considerably. The first of these effects is dispersion in the ISM that results in higher radio frequencies arriving earlier than the lower at the telescope which smears the pulse. Secondly, the intrinsically narrow pulse is broadened due to multipath propagation effects (e.g. Williamson 1973). Past studies at low frequencies were limited either by insufficient removal of dispersion or low sensitivity of observing systems and hence resulted in fewer giant pulses being detected at low frequencies. For example, the early studies relied on fewer than 400 giant pulses which were also not coherently dedispersed (e.g. Sutton et al. 1971, at $160 \mathrm{MHz}$; and both Argyle \& Gower 1972; Gower \& Argyle 1972, at $146 \mathrm{MHz}$ ). More recently, Popov et al. (2006b) used a sensitive telescope, but incoherent dedispersion of the pulsar signal resulted in only 128 detected giant pulses at $111 \mathrm{MHz}$. Similarly, Bhat et al. (2007) found 31 pulses at $200 \mathrm{MHz}$ by coherently dedispersing the pulsar signal, but used a less sensitive system with an effective area, $A_{\mathrm{eff}} \approx 50 \mathrm{~m}^{2}$. The need for coherent dedispersion is highlighted by the $\sim 23$ pulse periods or $766.75 \mathrm{~ms}$ of dispersion smearing within the $2.5 \mathrm{MHz}$ band at $116.75 \mathrm{MHz}$, which is the lowest band in our observations. Even with a reasonable 64-channel hardware filter bank, the dispersion smearing in each channel is $11.9 \mathrm{~ms}$, which if not removed allows the detection of only the very bright pulses.

The Crab pulsar has a rather steep spectrum with a spectral index $\alpha=-3$ (Rankin et al. 1970; Maron et al. 2000). This implies that a large number of giant pulses should be detectable at low radio frequencies, if their occurence rate remains the same as that reported at higher frequencies (Lundgren et al. 1995). Scattering in the ISM affects normal pulses and giant pulses alike, but the effect is more easily measured in the intense giant pulses owing to their narrowness. If the dispersion is fully 
Table 1. Observation details.

\begin{tabular}{ccccc}
\hline \hline $\begin{array}{c}\text { Frequency } \\
(\mathrm{MHz})\end{array}$ & $\begin{array}{c}\text { Session I } \\
08: 59: 50^{a}\end{array}$ & $\begin{array}{c}\text { Session II } \\
11: 59: 50^{a}\end{array}$ & $\begin{array}{c}\text { Session III } \\
14: 59: 50^{a}\end{array}$ & $\begin{array}{c}\text { Session IV } \\
17: 59: 50^{a}\end{array}$ \\
\hline $116.75 \ldots$ & $\mathrm{X}$ & $\mathrm{X}$ & $\mathrm{X}$ & $\mathrm{X}$ \\
$122.75 \ldots$ & $*$ & $*$ & $*$ & - \\
$139.125 \ldots$ & $\mathrm{X}$ & $\mathrm{X}$ & $\mathrm{X}$ & - \\
$141.75 \ldots$ & - & - & $\mathrm{X}$ & $\mathrm{X}$ \\
$147.50 \ldots$ & - & $\mathrm{X}$ & $\mathrm{X}$ & $\mathrm{X}$ \\
$157.00 \ldots$ & $\mathrm{X}$ & $\mathrm{X}$ & $\mathrm{X}$ & $\mathrm{X}$ \\
$162.50 \ldots$ & $\mathrm{X}$ & $\mathrm{X}$ & $\mathrm{X}$ & - \\
$173.85 \ldots$ & $\mathrm{X}$ & $\mathrm{X}$ & $\mathrm{X}$ & $\mathrm{X}$ \\
\hline
\end{tabular}

Notes. ${ }^{(a)}$ Observation start times of the 15 -min sessions in UTC. (b) Successful runs are marked "X", runs that failed with "-_" and sessions severely affected by RFI with “*”.

corrected, the giant pulse emission rates and the scattering phenomena can be studied in much greater detail. The observing system we use has the possibility to coherently dedisperse the pulsar signal. Thus this study marks the first coherently dedispersed study of the emission from the Crab pulsar with a resonably sensitive telescope, resulting in a large number of giant pulses in the 110-180 MHz frequency range. The preceeding discussion motivates the revisit of the Crab giant pulse emission at low radio frequencies.

The rest of the paper is organised as follows. The observations are described in Sect. 2. The flux calibration, average radio emission and the spectra of the Crab pulsar are discussed in Sect. 3. Scattering in single pulses and across the frequency range is treated in Sect. 5. The single pulse statistics are discussed in Sect. 4, followed by discussion and conclusions.

\section{Observations and data reduction}

The observations reported here were carried out at the Westerbork Synthesis Radio Telescope (WSRT) using the Low Frequency Front Ends (LFFEs) on 13 May 2006 (for details, see Table 1). The pulsar signal was recorded and processed using the PuMa-II instrument (Karuppusamy et al. 2008). The LFFEs were tuned to eight frequencies each of which was $2.5 \mathrm{MHz}$ wide in the 110-180 MHz frequency range and the signals were recorded to disk. The recorded signal was spread over four 15min sessions (see Table 1). This baseband data was then coherently dedispered using the dispersion measure (DM) obtained from the Crab pulsar ephemeris maintained by the Jodrell Bank Observatory $^{1}$. A 32-channel coherent filterbank was formed using the open-source pulsar data processing software package DSPSR (van Straten \& Bailes 2011) and all single pulses were written to disk. The PSRCHIVE software (Hotan et al. 2004) was used to form total intensities and for further processing of the data. Single pulses satisfying the threshold $S / N \geq 4.4 \sigma$ in total intensity were examined by eye and pulses not contaminated by RFI were retained for the single pulse analysis.

The average pulse profiles were also formed every 10 -s using a 64-channel coherent filterbank. The averaged emission from the pulsar in all four sessions at two frequencies are displayed as a grey scale plot in Fig. 1. The system was more sensitive in two of the four sessions because the sensitivity of the WSRT to the pulsar depends on the hour angle of the Crab nebula (explained in the following section).

\footnotetext{
${ }^{1}$ http://www.jb.man.ac.uk/ pulsar/crab.html
}

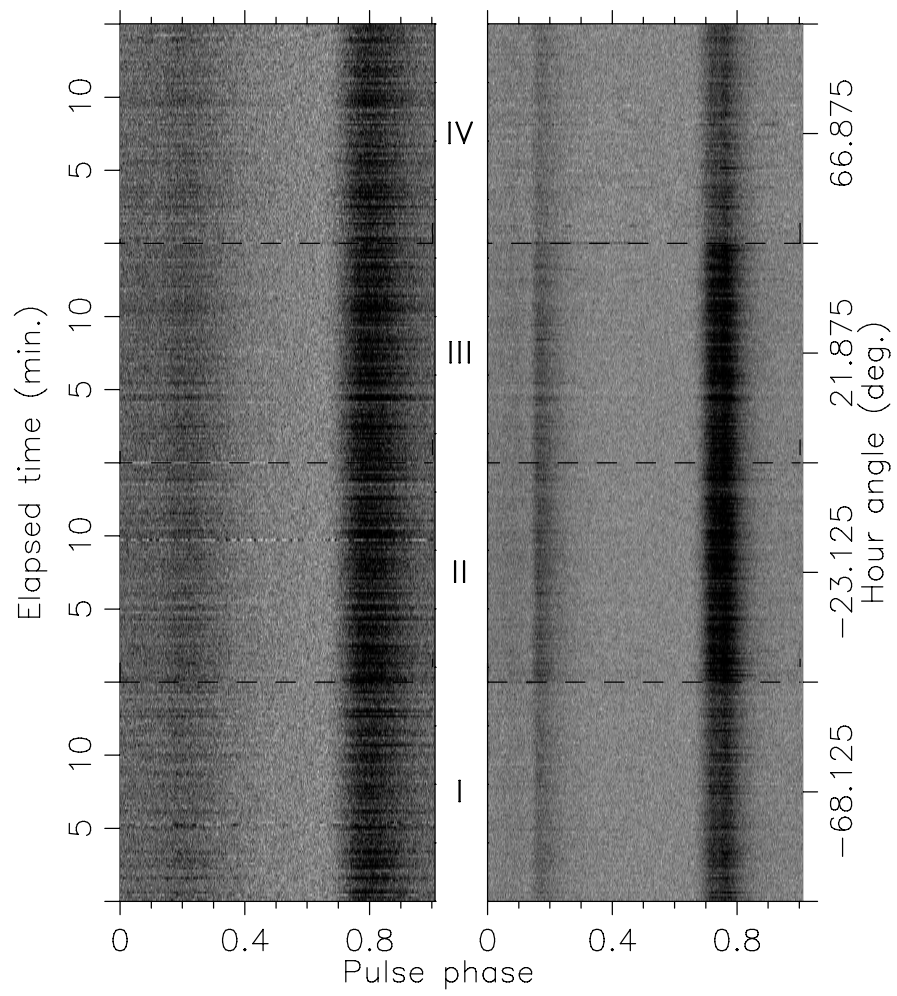

Fig. 1. Plot of total intensity of Crab pulsar against time in the four observing sessions shown separated by dashed horizontal lines. The left panel corresponds to the band centred at $116.75 \mathrm{MHz}$, while the panel on the right is at $157 \mathrm{MHz}$. The elapsed time shown on the vertical axis is the observation time within each session. The vertical axis is labelled with the hour angle of the Crab pulsar on the right side of the second panel and corresponds to the middle of the 15-min observing sessions.

\section{Radio flux of the Crab pulsar}

\subsection{Flux calibration}

The Crab nebula is a bright extended object with a typical size of $6^{\prime} \times 4^{\prime}$ and therefore dominates the telescope system temperature when the width of the telescope beam is comparable to the angular size of the nebula. The frequency dependence of the nebular flux can be expressed as $955 v^{-0.27} \mathrm{Jy}$, where $v$ is the frequency in $\mathrm{GHz}$ (Bietenholz et al. 1997). For these observations, the WSRT was configured to operate in the tied array mode, where the signals from fourteen 25-m telescopes were coherently added by purpose-built hardware. The resulting synthesised beam depends on the source hour angle and radio frequency. In the 110$180 \mathrm{MHz}$ range the beam is smaller than the nebula at higher frequencies and at small hour angles of the Crab pulsar. On the other hand, at $116 \mathrm{MHz}$ the beam is approximately the size of the nebula and the emission from the nebula is unresolved. This increases the system temperature and reduces system sensitivity. The resulting pulse intensity variation with respect to observation time is displayed in Fig. 1 and shows the frequency-time dependence of the beam width. From the figure, it is clear that the signal-to-noise ratio $(S / N)$ in sessions II and III are larger than the other two sessions at $157 \mathrm{MHz}$. Refering to Table 1, session III contains more data than session II. Therefore, we use the average profiles formed from the observing session III to estimate the average pulse flux.

Observations at low radio frequencies are prone to RFI and the data affected by RFI has to be removed before calibration of the single pulses or the average pulse profile. To clean RFI in 
R. Karuppusamy et al.: Crab giant pulses at low frequencies

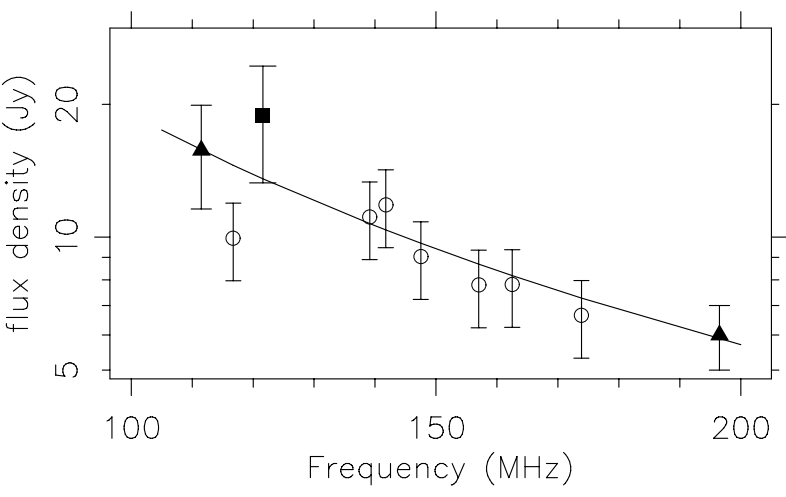

Fig. 2. The spectrum of the Crab pulsar in the 110-200 MHz range. Solid triangles represent flux densities at 111.5 and $196.5 \mathrm{MHz}$, respectively (Vandenberg et al. 1973). Flux density at $121.6 \mathrm{MHz}$ (shown using filled square) comes from Erickson et al. (1972). Open circles are fluxes derived from observing session III of the present work. The error bars on the open circles correspond to $20 \%$ uncertainty in the flux measurements.

the average profiles, the 10-s subintegrations were inspected visually as frequency-phase plots and RFI affected channels were removed in this process. The remaining data were integrated to estimate the average pulse fluxes.

The change in the nebular contribution to the system temperature $\left(T_{\text {sys }}\right)$ due to the frequency-time dependence of the beam width was incorporated in the estimation of pulse fluxes in a manner similar to Sect. 3 of Karuppusamy et al. (2010). In short, the increase in $T_{\text {sys }}$ due to the Crab nebula was multiplied by a factor that is a function of radio frequency and the hour-angle of the Crab pulsar. The aforementioned work was done at $1400 \mathrm{MHz}$ and we use values corresponding to the frequency range considered here. Accordingly, the receiver temperature is taken to be $400 \mathrm{~K}$ in the $110-180 \mathrm{MHz}$ range of the LFFE. The nominal background sky temperature spectrum of $v^{-2.6}$ was compensated electronically in the amplitude gains of LFFE amplifiers. To this value, the contribution of the Crab nebula is added to give the system temperature, assuming a $25 \%$ aperture efficiency and a telescope gain of $0.57 \mathrm{~K} \mathrm{Jy}^{-1}$. The uncertainty in the flux estimates are taken to be $20 \%$, which includes the possible effects due to the remaining RFI and the uncertainties in the estimation of the Crab nebula's contribution to the system temperature.

\subsection{Radio spectrum}

The flux calibrated average pulse profiles from the observing session III were used to compute the mean continuum flux density for the Crab pulsar because this was the session least affected by RFI and therefore has the largest $S / N$ of the four observing sessions. The spectrum is displayed in Fig. 2 and is derived from the computed mean flux densitites. For comparision, previously published pulsed flux densities at $111.5,121.6$ and $196.5 \mathrm{MHz}$ are included in this figure. The pulsed signal strength 111.5 and 121.6 MHz was computed by substracting the steady emission from the nebula (details in Table 2). A power law with an index of $-1.73 \pm 0.45$ results in good fit for the spectra displayed in Fig. 2.

Two features in this figure are noteworthy. The flux densities from our work are somewhat lower than values published earlier. Second, the power law fit to the spectrum here is shallower than the value -2.5 reported by Erickson et al. (1972).
Table 2. Crab pulsar flux densities.

\begin{tabular}{cccc}
\hline \hline $\begin{array}{c}\text { Frequency } \\
(\mathrm{MHz})\end{array}$ & Flux ratio $^{a}$ & $\begin{array}{c}\text { Total flux } \\
\text { Jy }\end{array}$ & $\begin{array}{c}\text { Pulsed flux } \\
\text { Jy }\end{array}$ \\
\hline $111.5^{b} \ldots$ & $0.45 \pm 0.10$ & $35 \pm 5$ & $15.75 \pm 4.16$ \\
$121.6^{c} \ldots$ & $0.65 \pm 0.15$ & $29 \pm 6$ & $18.85 \pm 5.57$ \\
$196.5^{b} \ldots$ & - & $6 \pm 1$ & $6 \pm 1$ \\
\hline
\end{tabular}

Notes. ${ }^{(a)}$ This is the ratio of steady emission from nebula to the pulsed emission from the pulsar. ${ }^{(b)}$ From Vandenberg et al. (1973). ${ }^{(c)}$ From Erickson et al. (1972).

Possible reasons for this departure could be an underestimation of the system temperature or an overestimation of gain in our work. In addition, our flux density estimates are based entirely on the pulsed data. In contrast, VLBI techniques were used in the aforementioned work in which the pulsar was treated as a compact source. Hence any unmodelled scattered emission results in a DC offset in the flux density estimates, which are not detected in our observations. The combination of the above factors may lead to the discrepancy seen in our observations.

\subsection{Average pulse profiles}

Figure 3 shows the average emission profile at several frequencies observed at the WSRT and at $\gamma$-ray wavelengths from the Fermi-LAT (Abdo et al. 2010). It is of interest to note the features in the average emission at the low sky frequencies. The increase in scatter broadening of the pulses is clearly visible as one moves down in frequency. At least four emission components are seen - the precursor to the mainpulse, the main- and interpulse and the precursor to the interpulse (see Fig. 3). A weak emission brigde that appears to connect the main- and interpulse appears convolved with the scatter tail of the mainpulse. These emission features are discussed below. In the $1400 \mathrm{MHz}$ data, the weak emission that preceeds the mainpulse is the low-frequency component (Fig. 1; Moffett \& Hankins 1996).

In addition to scatter broadening of the average emission components, the ratio of main- to interpulse peak intensity gradually decreases with the increase in frequency. The emission bridge is convolved with the mainpulse scatter tail and it increases towards lower frequencies. At $116.75 \mathrm{MHz}$ this feature is $\sim 40 \%$ of the peak mainpulse intensity. Previous studies have revealed the presence of an emission bridge (Rankin et al. 1970; Manchester et al. 1972; Vandenberg et al. 1973), although it was not very evident as the signals were affected by dispersion smearing.

Scattering renders the precursor to the mainpulse unresolvable below $147.75 \mathrm{MHz}$ but vestiges of it are still visible at $139.75 \mathrm{MHz}$. The intensity of the precursor peaks at $173.85 \mathrm{MHz}$, and shows a steep spectrum. The precursor is fully resolved at $382 \mathrm{MHz}$, and at $608 \mathrm{MHz}$ but much weaker in intensity. The phase separation between the mainpulse and the precursor appears to show a marginal increase with frequency. In order to examine any trends in this separation, the average profiles at each frequency were modelled using up to 5 Gaussians components. The results from the fits is consistent with no frequency dependent change in the longitudinal separation of the precursor and the mainpulse. This further confirms that the precusor and the LFC component are distinct as pointed out by Moffett \& Hankins (1996).

In all low frequency average emission profiles in Fig. 3 indications of a precursor to the interpulse is seen. At $116.75 \mathrm{MHz}$ this component is almost masked by the long scatter tail of the 


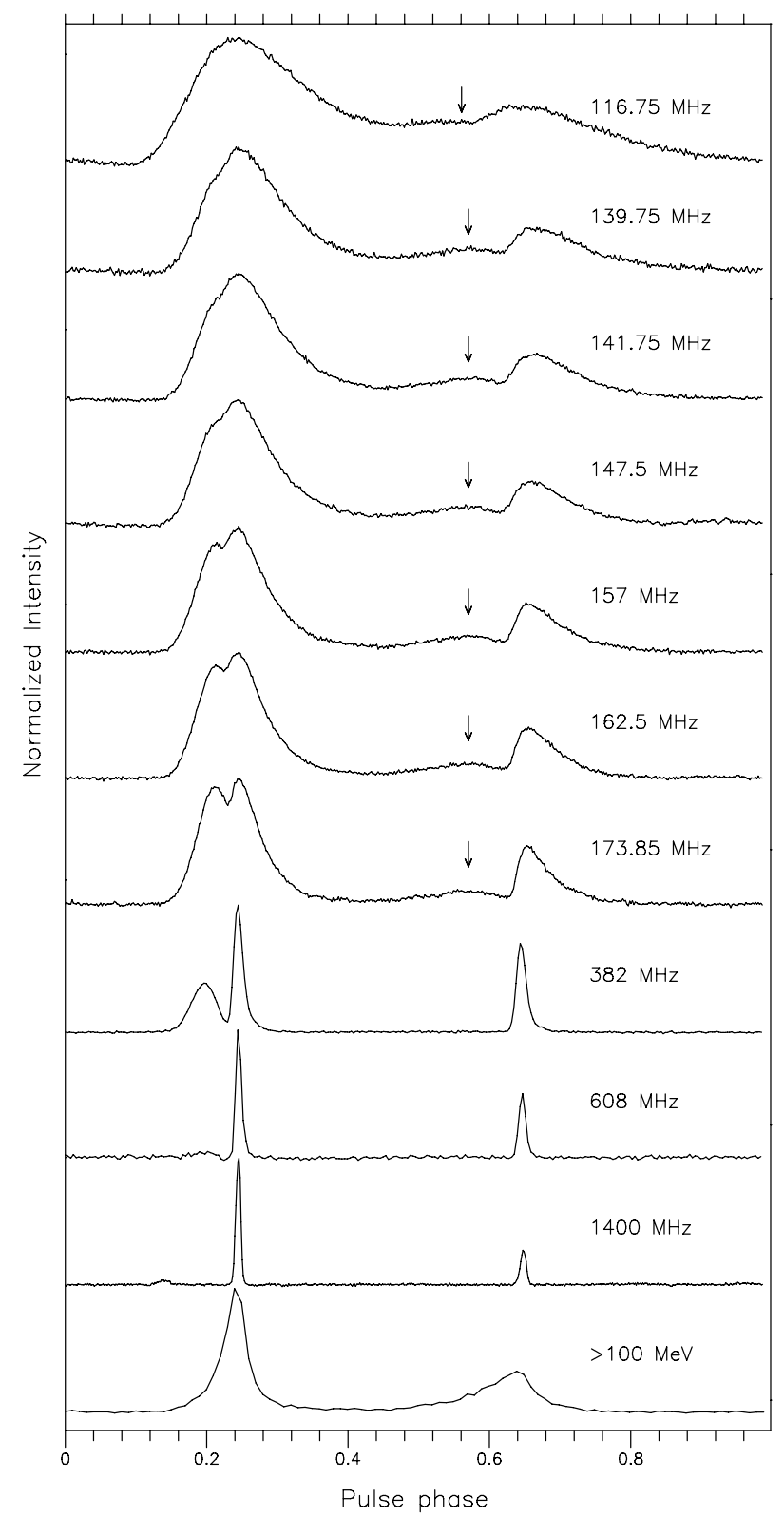

Fig. 3. Plot showing the average emission profiles of the Crab pulsar at several frequencies observed at the WSRT arbitrarily aligned by the mainpulse peak. The first seven profiles are from the observations reported in this work, while profiles at 382,608 and $1400 \mathrm{MHz}$ are from the WSRT archives. The $\gamma$-ray profile is from Abdo et al. (2010). The arrow shows the location of the interpulse precursor emission component.

mainpulse. However, as indicated by the small arrow in the figure, the slight increase in emission is clearly visible. This component is also visible in the early models of the scattered emission from the pulsar (Counselman \& Rankin 1971; Manchester et al. 1972) and was detected by Moffett \& Hankins (1996). With our improved sensitivity and resolution, we confirm the presence of the precursor to the interpulse at these frequencies. We now draw comparision to the mainpulse counterpart. Both features appears to increase in intensity as we move down in frequency. These components have no emission counterparts at higher radio frequencies or other wavelengths. Other than the first order comparision, more observations are necessary to ascertain the nature of these components. For example, the mainpulse precursor is linearly polarized (Campbell \& Heiles 1970) and its intensity varies in time on the order of a few tens of minutes (Rankin et al. 1970). Our observations show that the precursor to the interpulse is devoid of any giant pulse emission and therefore consists of normal pulses only. This is similar to the mainpulse precursor (Popov et al. 2006a) and is discussed in Sect. 6.

The pulsed $\gamma$-ray emission appears to be coincident with the radio giant pulse emission phases, although high-time resolution data shows that the $\gamma$-ray peak leads the radio peak by 0.002 in phase (Abdo et al. 2010). There are no other high-energy counterparts to the emission features discussed above.

\section{Giant pulse statistics}

In this section, the statistics of the giant pulses are examined using the mean flux density distribution of single pulses. We began by searching for the giant pulses based on a threshold of $S / N \geq 4.4 \sigma$, where $\sigma$ is the off-pulse root-mean-square variation of the noise. Furthermore, only pulses that showed proper phase association to either the two precursors, the main- or the interpulse components of the average emission profile were collected. Next, the flux density of single pulses was computed, and then averaged over the pulse period to compute the average flux density of the single pulses. At low radio frequencies the scattered giant pulses can occupy a considerable fraction of the pulse period. For this reason, a few giant pulses were visually examined in every band recorded, following which a window of suitable size was chosen to include all pulsed emission from the star. After calibrating the pulses, the flux was integrated in this window and divided by the peak value resulting in the equivalent width, $W_{\text {eq }}$ of the pulse. The flux of the pulse is then computed using $W_{\text {eq }}$ in the radiometer equation (Sect. 3; Karuppusamy et al. 2010) and then averaged over the pulse period,

$W_{\mathrm{eq}}=\frac{1}{I_{\max }} \times \sum_{\mathrm{i}=\mathrm{n}_{1}}^{\mathrm{n}_{2}} I_{\mathrm{i}} \times 4.1 \mu \mathrm{s}$,

subsequently, a second level search was undertaken to locate all broad band pulses. Using the giant pulses at $173.85 \mathrm{MHz}$ and the dispersion delay at other frequencies, simultaneous pulses were recovered. This resulted in pulses at $173.85 \mathrm{MHz}$ and at least one other frequency. 16 of the 172 giant pulses detected at $173.85 \mathrm{MHz}$ were also detected in the other seven bands with $S / N>15 \sigma$. The pulses are very broad band, i.e $\Delta v / v \sim 0.3$, where $\Delta v /$ is the bandwidth. However, these pulses form a small fraction of the total number of giant pulses detected at $173.85 \mathrm{MHz}$, and we now show that scintillation cannot be a possible cause. The diffractive scintillation bandwidth, $\Delta v_{\mathrm{d}}$ at $430 \mathrm{MHz}$ for the Crab pulsar was found to be $24 \mathrm{kHz}$ (Table 3; Cordes et al. 2004). Using a nominal $\Delta v_{\mathrm{d}} \propto v^{4}$ frequency scaling law, we expect $\Delta v_{\mathrm{d}}$ to be on the order of $0.12-$ $8 \mathrm{kHz}$ and this is much smaller than the $2.5 \mathrm{MHz}$ bandwidth used. Therefore, diffractive scintillation cannot be the cause for fewer broad band giant pulses. Also, refractive interstellar scintillation cannot cause deep intensity modulations across the frequency band observed as the timescales are on the order of a few days (Lundgren et al. 1995). The foregoing discussion illustrates that the scintillation could not have limited the number of broadband giant pulses detected and such emissions are relatively rare events.

No single pulses were detected at phases of the two precursor components. The results of the remaining giant pulse detections are shown in Table 3 and these correspond to the phases of the main- and interpulse. The reason for a larger number 
Table 3. Properties of the Crab giant pulses.

\begin{tabular}{|c|c|c|c|c|c|c|}
\hline \multirow{2}{*}{$\begin{array}{c}\text { Frequency } \\
(\mathrm{MHz})\end{array}$} & \multicolumn{2}{|c|}{${ }^{a} N_{\text {pulses }}$} & \multirow[t]{2}{*}{$N_{\text {total }}$} & \multirow[t]{2}{*}{${ }^{b} \alpha_{\mathrm{mp}}$} & \multirow[t]{2}{*}{${ }^{b} \alpha_{\mathrm{ip}}$} & \multirow{2}{*}{$\begin{array}{r}{ }^{c} \text { Rate }\left(\times 10^{-3}\right) \\
\mathrm{s}^{-1}\end{array}$} \\
\hline & MP & IP & & & & \\
\hline $116.75 \ldots$ & 1246 & 370 & 109090 & $2.12 \pm 0.09$ & $2.70 \pm 0.29$ & $14.81 \pm 0.37$ \\
\hline 139.125 & 581 & 206 & 81818 & $1.65 \pm 0.14$ & $1.20 \pm 0.13$ & $9.62 \pm 0.34$ \\
\hline 141.75 . & 964 & 156 & 54545 & $1.51 \pm 0.05$ & $1.60 \pm 0.09$ & $20.53 \pm 0.61$ \\
\hline 147.50 & 1047 & 313 & 81818 & $1.78 \pm 0.10$ & $1.60 \pm 0.12$ & $16.62 \pm 0.45$ \\
\hline 157.00 & 1175 & 409 & 109090 & $1.83 \pm 0.10$ & $0.70 \pm 0.05$ & $14.52 \pm 0.36$ \\
\hline 162.50 & 1165 & - & 81818 & $2.38 \pm 0.15$ & - & $14.23 \pm 0.42$ \\
\hline 173.85 & 1355 & 330 & 109090 & $2.39 \pm 0.12$ & $2.05 \pm 0.16$ & $15.45 \pm 0.38$ \\
\hline
\end{tabular}

Notes. ${ }^{(a)}$ Number of giant pulses detected at the main- and interpulse phases. ${ }^{(b)}$ Slopes of the average pulse flux histograms for the main- and interpulse giants. ${ }^{(c)}$ Computed rate is based on all detected giant pulses. The error on the rate is $\sqrt{N_{\text {pulses }}} / N_{\text {total }}$ under the assumption that the occurence of giant pulses are Gaussian distributed.

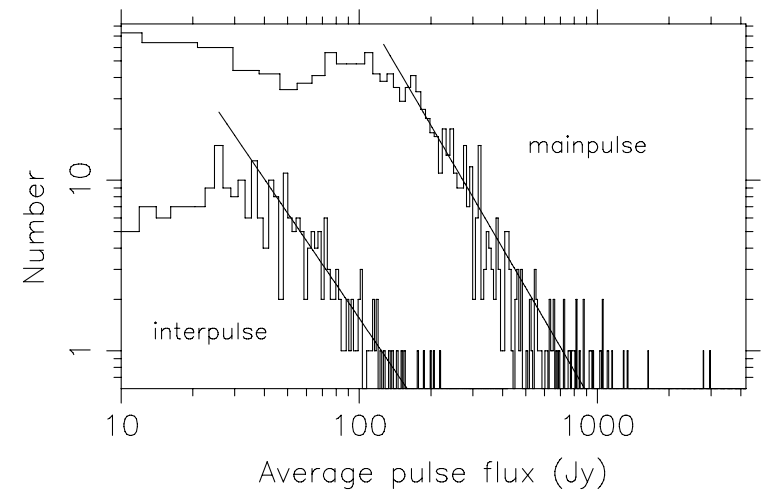

Fig. 4. Plot showing the distribution of the average flux density for the pulses found at the main- and interpulse phases at the band centred at 173.85 MHz. Giant pulses detected in other bands show a similar trend. Also shown are the power law fits to the distributions and the values are listed in Table 3.

of detections in the higher frequency bands is due to the increased sensitivity of the telescope array as discussed earlier in Sect. 3.1. At $116.75 \mathrm{MHz}$, a somewhat larger number of pulses were detected owing to the longer observation (see Table 1). The number of pulses detected in the bands centred at 139.125 and 141.75 MHz are considerably less than the other bands because only two of the four observing session contained usable data for these two bands.

Figure 4 shows the flux distribution of the giant pulses at 173.85 MHz. The other bands display similar histograms and are therefore not shown. In all bands we note that, the giant pulses are more frequent and bright at the mainpulse phase compared to those at the interpulse phase, and this is entirely consistent with the emission at higher frequencies (Lundgren et al. 1995). To compare the giant pulse emission in various bands we model emission with the probablity density function and it is expressed as,

$f\left(S_{v}\right) \propto S_{v}^{-\alpha}, \quad$ for $S_{v}>S_{b}$

where $S_{v}$ is the mean flux density at frequency $v$, and $S_{b}$ defines the point in the distribution beyond which a power law emerges. Using a similar method and as displayed in Fig. 4, power-law fits to the pulse flux distributions at other frequencies were made, and the slopes derived are shown in Table 3. The slopes of the distributions steepen with frequency except at 116.75 MHz, which is probably due to an uncertainty in estimation of average pulse flux. Additionally, in this band the pulse emission occurs over a large fraction of the pulse period resulting in an insufficient off-pulse region contributing to flux estimation uncertainties. Alternatively, the drastic turn over in the spectrum of the pulsar at $\sim 100 \mathrm{MHz}$ (Rankin et al. 1970; and Fig. 2 in Sect. 3.2) might cause a steepening in the distributions.

The slopes of the flux distributions found here can be compared to those reported elsewhere. Argyle \& Gower (1972) report a slope of -2.5 and -2.8 for the main- and interpulse flux distributions at $146 \mathrm{MHz}$. These results are based on their 171 pulses in two observations using 46-m and 26-m antennae. The difference in the values we derive may arise from the small number statistics and limited sensitivity of Argyle \& Gower (1972). We can also compare the slopes estimated at other frequencies. Karuppusamy et al. (2010) report slopes of -3.0 and -2.8 at $1400 \mathrm{MHz}$ for the main- and interpulses flux distributions, respectively. Similarly, Lundgren et al. (1995) find a value of -3.3 at $800 \mathrm{MHz}$ and Cordes et al. (2004) report $\approx-2.3$ at $430 \mathrm{MHz}$ and noted that the slope increased with frequency. Combined with our values at $110-180 \mathrm{MHz}$, we confirm the general steepening of the flux distributions with increasing frequency. The emission rate of giant pulses is also higher at low frequencies. At $173 \mathrm{MHz}$, a total of 1685 giant pulses above with $S / N \geq 5.2 \sigma$ were detected (see Table 3 ). This translates to a rate of one giant pulse in 62 stellar rotations. The giant pulse emission rate (Col. 6 in Table 3 ) shows a slight increase with decreasing frequency, except for the bands at 116.75 and $139.125 \mathrm{MHz}$. At these frequencies, the complex nature of the telescope beam and the presence of any residual RFI might have affected the derived giant pulse rates.

As reported elsewhere, we confirm that there are no giant pulses emitted at phases other than that of the average main- and interpulse emission. An extensive search at both the precursor phases resulted in no single pulse detections. Furthermore, we computed the longitude resolved modulation index (Weltevrede et al. 2006) in the longitude range corresponding to the mainpulse precursor, and find a value of $\approx 0.8$. The large number of giant pulses at the main- and interpulse phases resulted in strong intensity variations leading to a correspondingly large modulation index of $\approx 3$. This supports the argument that the mainpulse precursor consists of entirely normal pulses, and hence shows a very small pulse to pulse intensity variation, unlike the emission at the main- and interpulse phases.

\section{Scattering analysis}

\subsection{Scatter timescales}

Single bright pulses are excellent probes of the scattering ISM. It is known that for frequencies above $1 \mathrm{GHz}$ single pulse emission may contain more than one component resulting in "microbursts" (Crossley et al. 2010). However, at low frequencies, the propagation of the intrinsically narrow pulse through the ISM 


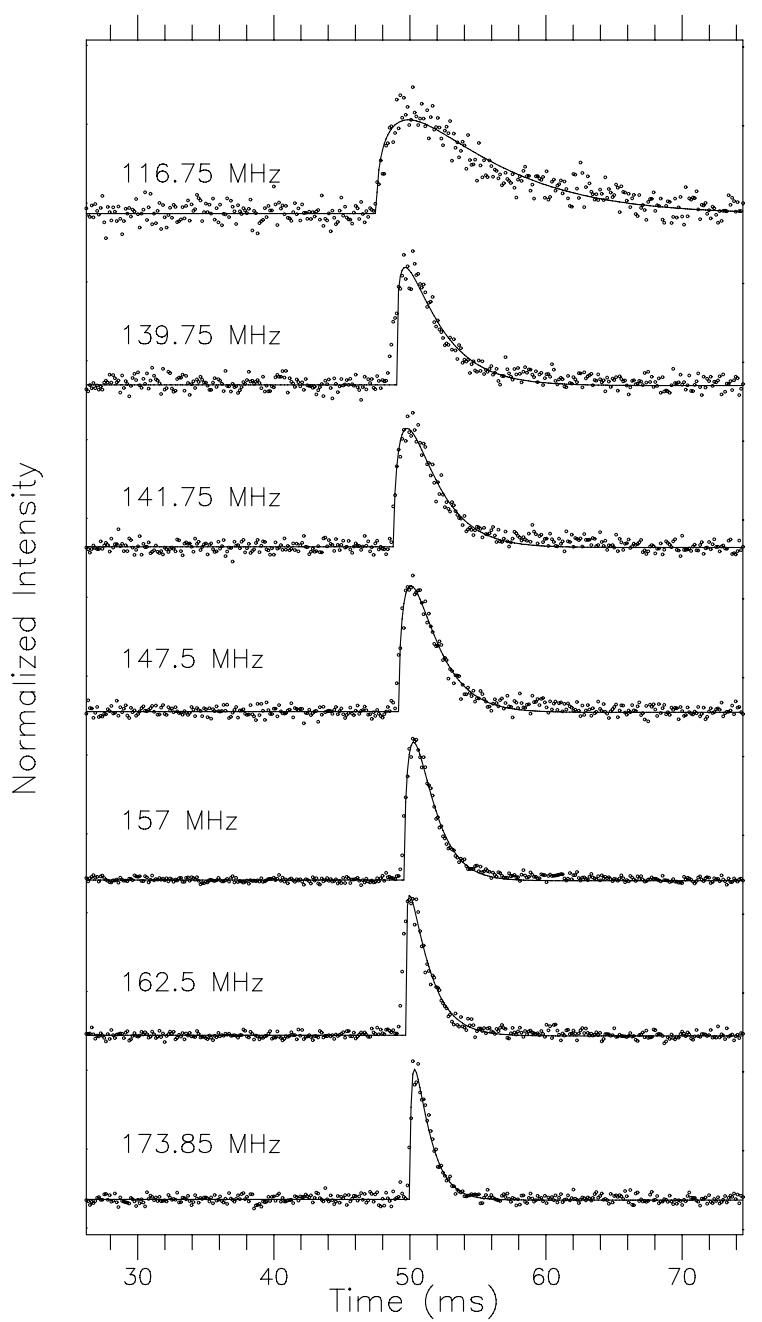

Fig. 5. Plot displaying a broad band giant pulse detected in seven of the eight recorded bands. The observed total intensity of the pulse is modelled using a functional form described in the text.

and the scattering material within the Crab nebula results in a single, smooth exponential tail in the average profiles and giant pulses. We model the giant pulses as an asymmetric exponential function and is written as,

$f(t)=\lambda \cdot\left(t-t_{\mathrm{m}}\right)^{\beta} \cdot \mathrm{e}^{-\frac{\left(t-t_{\mathrm{m}}\right)}{\tau_{s c}}}$

where $t_{\mathrm{m}}$ is the time when the exponential decay in intensity begins, $\lambda$ and $\beta$ are constants. Usually, $\beta$ is set to unity which results in a simple functional form that has a fast-rise and an exponential decay (Eilek et al. 2002). However, at low frequencies this results in a bad fit and therefore the pulse can no longer be described by this model. For instance, a thick slab of scattering material near the source can give rise to a comparatively slow rise and a shallow decay of the pulse (Williamson 1972). Therefore, in our work, the parameter $\beta$ is also derived from the model fits and is found to lie in the range $0.19 \pm 0.03$ to $0.99 \pm 0.18$ for the 116.75-173.85 MHz frequency range. The best fit curves to a few pulses based on Eq. (3) are shown in Fig. 5. The scattering timescales $\tau_{\mathrm{sc}}$ are given by the $1 / e$ decay times and are estimated from the exponential fits to the trailing part of the giant pulses.

If the scattering in the direction of the Crab pulsar is assumed to originate from a single, thin slab of scattering material giving rise to a Kolgomorov spectrum, then this results in a frequency scaling law $v^{-4.4}$. To test this model, the average of the derived
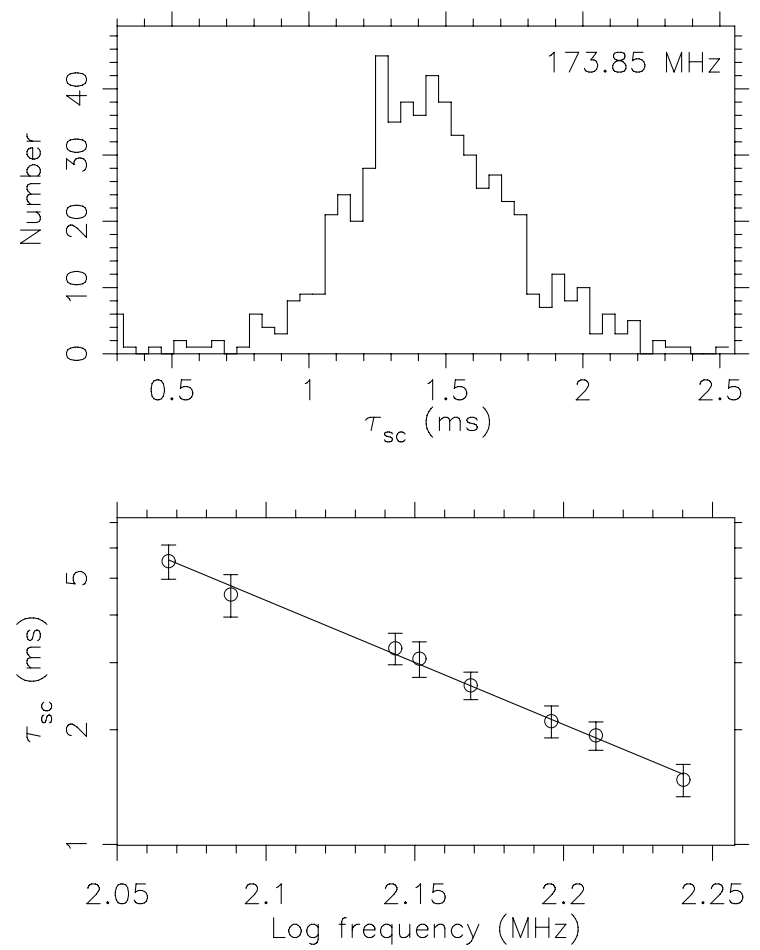

Fig. 6. Plot showing scatter times from the model fits. The top panel shows the distribution of the scattering timescales in the band centred at 173.85 MHz. The bottom panel shows the average scattering timescales from the other bands.

scatter times from the individual giant pulses in every observed band was computed. Example fits are displayed for seven of the observed bands in Fig. 5.

\subsection{Statistics of scatter timescales}

The distribution of the scatter times is displayed in the top panel of Fig. 6 and corresponds to the band centred at 173.25 MHz. Giant pulses in bands at other frequencies observed here display qualitatively similar histograms. The spread in the histogram is caused by the values from fits to weak pulses, which may have larger errors. The average of the scattering timescales was computed at each frequency. These values are plotted and the best fit is shown in the lower panel of Fig. 6. The spectrum of scatter timescales is well-described by a $\tau_{\mathrm{sc}} \propto v^{-3.2 \pm 0.08}$ relation. At an epoch only 233 days earlier than our observations, Bhat et al. (2007) derive a frequency scaling of $v^{-3.5 \pm 0.3}$ based on $\tau_{\mathrm{sc}}$ at several frequencies reported elsewhere and their value at $200 \mathrm{MHz}$. The Crab nebula also shows abnormal scattering events where scattering timescales can drastically change with in a few weeks (Kuzmin et al. 2008). Recent observations with LOFAR and the giant pulses detected in the radio band similar to this study, have shown scattering times larger than 10 times the value reported here (Stappers et al. 2011). Considering this, the frequency scaling we derive is in good agreement with that of Bhat et al. (2007). The lower index of $\sim-3.2$ to -3.5 is inconsistent with the expectation of -4.4 for a Kolgomorov spectrum. However, the Kolgomorov spectrum is applicable to the distributed ISM, while most of the scattering in the pulses from Crab pulsar orginates within the nebula (Lyne et al. 2001).

\section{Discussion and conclusion}

The simultaneous average emission profiles at several frequencies in the 110-180 MHz range are reported for the first time. 
From the coherently dedispersed signal, we confirm the previously identified interpulse precursor and we compared it to the mainpulse precursor. Popov et al. (2006a) hypothesise the latter emission component as consisting of normal pulses from the pulsar, and that the conventional mainpulse is made of entirely giant pulses. Their arguments were based on the finding that the average profile formed using only giant pulses has a remarkable similarity to the average emission profile at that frequency and that the phase of giant pulses trail the average emission in certain pulsars like PSR B1937+21 (Kinkhabwala \& Thorsett 2000). This interpretation was supported by the high-energy emission occuring coincident with the phase of the giant pulses as seen in PSRs B1937+21 (Cusumano et al. 2003), B1821-24 (Romani \& Johnston 2001) and the Crab pulsar (Fig. 3). The foregoing discussion and the interpulse precursor support the view of Popov et al. (2006a) where the conventional main- and interpulse emission consists only of giant pulse emission.

The interpulse precurcor component can be robustly tested by means of a simulation and further observations. This can be done, for example, by assuming a $x \mathrm{e}^{-x}$ functional form of the scattering effects, and a narrow pulse representing the pulse emitted at the source. A model with an interpulse precursor emission component was tested in the work of Manchester et al. (1972), and this template was smoothed using functions that represent both dispersive and scattering effects. With our observations, the dispersive effects are completely removed, and only the effects of scattering needs to be tested. We note that the precursor components show a large variation below $350 \mathrm{MHz}$. Observations in the $160-240 \mathrm{MHz}$ radio band with LOFAR (Stappers et al. 2011) can therefore shed more light on these emission features. Combined with observations at other sky frequencies, we further confirm that the main- and interpulse precursor components have very steep spectral indicies. Moreover, these components have no counter parts at other wavelengths. The radio spectrum of the Crab pulsar we derive is consistent with values published in earlier works.

The Crab giant pulse emission is limited to the main- and interpulse phases at this frequency, as reported in past studies. The phase of the main- and interpulses has counter parts at $\gamma$ ray wavelengths. Shearer et al. (2003) report enhanced emission at optical wavelengths coincident with the radio giant pulses. However, this is not seen at $\gamma$-ray wavelengths at either $800 \mathrm{MHz}$ (Lundgren et al. 1995) or at $8.9 \mathrm{GHz}$ (Bilous et al. 2011). There has been one reported detection of giant pulse emission at the so-called high frequency component (Jessner et al. 2005), at a frequency of $8.35 \mathrm{GHz}$. Even though we detect the precursor component with a large $S / N$, we confirm that no giant pulses are detected in the longitude corresponding to the precursor. A low value of modulation index at the precursor phase is a further evidence that the giant pulses donot occur at this phase or have an extremely low occurance rate.

Sallmen et al. (1999) report that the Crab giant pulses can very broadband from their simultaneous multi-frequency observations and find that $70 \%$ of pulses had $\Delta v / v \approx 0.8$ at $\sim 1 \mathrm{GHz}$. Our finding that some giant pulses with $\Delta v / v \approx 0.3$ suggests that the broad band nature of the giant pulse emission persists down to low radio frequencies. However, we note that only a small fraction of the detected giant pulses show such broadband emission in contrast to the vast majority of the pulses. From the general increase of the slopes of pulse flux distributions with frequency, it is clear that the giant pulses are more frequent at lower sky frequencies. Simultaneous multifrequency observations and similar data reduction and analysis techniques used here might help resolve the true emission bandwidths of giant pulses.

We have probed the propagation effects in the ISM and the Crab nebula in the $115-175 \mathrm{MHz}$ range from the scatter tails of the giant pulses. Complete removal of dispersion smearing allowed a greater experimental precision in the determination of scattering timescales than the previous studies. For the epoch of the observations reported here, the scattering time scales follow $v^{-3.2}$ dependence. The different values for the scattering spectrum derived by various authors confirms that a single Kolmogrov like spectrum is an insufficient description of the scattering medium. A possible reason for this departure is probably the nebula surrounding the pulsar.

Acknowledgements. We acknowledge the use of European Pulsar Data network, the ATNF pulsar catalogue and the SAO/NASA Astronomical Data System maintained by Harvard-Smithsonian Center for Astrophysics. The WSRT is operated by ASTRON/NWO. We thank the observers for the system set up and Roy Smits for help with observations. R.K. thanks M. Serylak for discussions and for help in data analysis. We thank the referee for the constructive comments. The PuMa-II instrument and R.K. were funded by Nederlands Onderzoekschool Voor Astronomie (NOVA).

\section{References}

Abdo, A. A., Ackermann, M., Ajello, M., et al. 2010, ApJ, 708, 1254 Argyle, E., \& Gower, J. F. R. 1972, ApJ, 175, L89

Bhat, N. D. R., Wayth, R. B., Knight, H. S., et al. 2007, ApJ, 665, 618 Bietenholz, M. F., Kassim, N., Frail, D. A., et al. 1997, ApJ, 490, 291

Bilous, A. V., Kondratiev, V. I., McLaughlin, M. A., et al. 2011, ApJ, 728, 110 Campbell, D. B., \& Heiles, C. 1970, Nature, 225, 527

Cordes, J. M., Bhat, N. D. R., Hankins, T. H., McLaughlin, M. A., \& Kern, J. 2004, ApJ, 612, 375

Counselman, C. C., \& Rankin, J. M. 1971, ApJ, 166, 513

Crossley, J. H., Eilek, J. A., Hankins, T. H., \& Kern, J. S. 2010, ApJ, 722, 1908

Cusumano, G., Hermsen, W., Kramer, M., et al. 2003, A\&A, 410, L9

Eilek, J. A., Arendt, Jr., P. N., Hankins, T. H., \& Weatherall, J. C. 2002, in Neutron Stars, Pulsars, and Supernova Remnants, ed. W. Becker, H. Lesch, \& J. Trümper, 249

Erickson, W. C., Kuiper, T. B. H., Clark, T. A., Knowles, S. H., \& Broderick, J. J. 1972, ApJ, 177, 101

Gower, J. F. R., \& Argyle, E. 1972, ApJ, 171, L23

Hankins, T. H., \& Eilek, J. A. 2007, ApJ, 670, 693

Hotan, A. W., van Straten, W., \& Manchester, R. N. 2004, Proc. Astron. Soc. Aust., 21, 302

Jessner, A., Slowikowska, A., Klein, B., et al. 2005, Adv. Space Res., 35, 1166 Karuppusamy, R., Stappers, B., \& van Straten, W. 2008, PASP, 120, 191 Karuppusamy, R., Stappers, B. W., \& van Straten, W. 2010, A\&A, 515, A36

Kinkhabwala, A., \& Thorsett, S. E. 2000, ApJ, 535, 365

Knight, H. S. 2007, MNRAS, 378, 723

Kuzmin, A., Losovsky, B. Y., Jordan, C. A., \& Smith, F. G. 2008, A\&A, 483, 13

Lundgren, S. C., Cordes, J. M., Ulmer, M., et al. 1995, ApJ, 453, 433

Lyne, A. G., Pritchard, R. S., \& Graham-Smith, F. 2001, MNRAS, 321, 67

Manchester, R. N., Huguenin, G. R., \& Taylor, J. H. 1972, ApJ, 174, L19

Maron, O., Kijak, J., Kramer, M., \& Wielebinski, R. 2000, A\&AS, 147, 195

Moffett, D. A., \& Hankins, T. H. 1996, ApJ, 468, 779

Popov, M., Soglasnov, V., Kondrat'ev, V., et al. 2006a, Astron. Lett., 50, 55

Popov, M. V., Kuz'min, A. D., Ul'yanov, O. M., et al. 2006b, Astron. Rep., 50, 562

Rankin, J. M., Comella, J. M., Craft, H. D., et al. 1970, ApJ, 162, 707

Romani, R., \& Johnston, S. 2001, ApJ, 557, L93

Sallmen, S., Backer, D. C., Hankins, T. H., Moffett, D., \& Lundgren, S. 1999, ApJ, 517, 460

Shearer, A., Stappers, B., O'Connor, P., et al. 2003, Science, 301, 493

Staelin, D. H., \& Reifenstein, III, E. C. 1968, Science, 162, 1481

Stappers, B. W., Hessels, J. W. T., Alexov, A., et al. 2011, A\&A, 530, A80

Sutton, J. M., Staelin, D. H., \& Price, R. M. 1971, in IAU Symp. 46, ed. R. D.

Davies, \& F. G. Smith (Reidel), 97

Vandenberg, N. R., Clark, T. A., Erickson, W. C., et al. 1973, ApJ, 180, L27

van Straten, W., \& Bailes, M. 2011, PASA, 28, 1

Weltevrede, P., Edwards, R. T., \& Stappers, B. W. 2006, A\&A, 445, 243

Williamson, I. P. 1972, MNRAS, 157, 55

Williamson, I. P. 1973, MNRAS, 163, 345 